\title{
Wind Power Fluctuation Characteristics of Wind Farms
}

\author{
Jing Zhang \\ School of North China Electric Power University, 071000, China \\ 965901765@qq.com
}

Keywords: Wavelet Neural Net, Sliding Difference Algorithm, Gentle Effect.

\begin{abstract}
The purpose of this paper is to research wind power fluctuation character of the wind farm. First, the qualitative and quantitative analysis methods are combined. Sliding difference algorithm is used to construct wind power fluctuations evaluation system. Calculating wind power fluctuations value volatility characteristics evaluation system at different time scales, we can get the conclusion that the optimum distribution form of distributed wind power fluctuations is t-location scale. Secondly, taking into account the wind power is non-linear, non-stationary characteristics of the data signal. This feature is fully in line with the wavelet neural net data analysis features. Therefore, the choice of wavelet neural network is learning and training and testing, and thus the total power of the future wind farms are amid to predict. And the research will predictive value as new learning data, for seven days after the wind power rolling forecasts. Then, comparative analysis of single units and the whole wind turbine power fluctuation characteristics obtained differences coverage under the volatility characteristic index wind power, and further understanding of volatility characteristic of wind power. The influence of temporal and spatial distribution and other factors, single wind turbine there is a big difference between the output power fluctuation characteristics of the wind farm, which is due to the presence of different turbine wind farm suffered difference, and wake effects between units, making the distribution of the frequent fluctuations in power. With respect to a single unit-He said fluctuation entire wind farm is more gentled, that with the increase in the spatial distribution of scale, fluctuating wind power presents a certain "Gentle Effect". Finally, the analysis overcome the adverse effects of wind power fluctuations on the grid operation, prepare the following specific solutions: rational allocation of energy storage devices, expanding the coverage area of the wind farm, or to improve the design of the windmill, so that wind farms can adapt to different directions wind, and you can try to eliminate the impact of fluctuations in the energy storage device by the wind farm output power to the grid, covering the area of large wind farms can adapt to different wind power complement each other, we have reached a stable amount of purpose into the grid.
\end{abstract}

\section{Introduction}

With increasingly stringent resource and environmental constraints, fossil fuel-based energy development model must be a fundamental change. Wind power does not consume any fuel, can be described as clean energy. Wind from the air campaign, not because of the development of wind power and exhausted, it is a renewable energy source.

Power wind turbines and wind speed main issue. Due to the impact of the wake wind between uncertainty and intermittent wind farm various units such as wind turbines based on the demand for electricity cannot be determined as a conventional power generators.

Large-scale wind power base is usually required to achieve access to the grid of transmission and wind power consumptive. Random fluctuations of wind power is considered to be the main factors adversely affect the grid. Fluctuation characteristics of wind power, whether to improve wind forecasting accuracy is of great significance to overcome the adverse effects of wind power on the grid access. 


\section{Setting up the Modeling}

\section{Wind power level S-level calculated volatility index.}

Dynamic test data in order to eliminate random fluctuations, use the point value of the function expressed its certainty of variation, you can use level sliding difference algorithm for processing test data.

Wind power fluctuation index basic formula:

$$
\left\{\begin{array}{l}
P_{f i}^{5 s}\left(t_{k}\right)=\left(P_{t_{k}-(N / 2-1)}+P_{t_{k}-(N / 2-1)}+\ldots+P_{t_{k}}+\ldots+P_{t_{k}+N / 2}\right) / N \\
P_{m i}^{5 s}\left(t_{k}\right)=P_{t_{k}}-P_{f i} \\
t_{k}=N / 2, N / 2+1, \cdots, N
\end{array}\right.
$$

\section{Wind farm power fluctuation characterization.}

Several common distribution describes the probability distribution of data as follows: normal distribution, non-parametric distribution, the logic Lancaster distribution, extreme value distribution, chi-square distribution. According to the present problem of stroke electric power characteristic time data, select the appropriate distribution of several of preselected distribution type. Analysis calculated value generator set features under different probability distributions.

Table 1 Characteristics of different distribution of wind turbines

\begin{tabular}{cccc}
\hline Probability distribution type & Means & Variance & $\begin{array}{c}\text { Standard } \\
\text { deviation }\end{array}$ \\
\hline t-location scale Distribution & 0.9217355 & 7635.2657 & 87.38001 \\
Normal Distribution & 0.9263955 & 7671.02 & 87.58436 \\
Logistic Distribution & -0.197532 & 4541.95 & 67.39399 \\
Extreme Distribution & -30.9858 & 28646.5 & 169.2527 \\
\hline
\end{tabular}

Qualitatively drawn: with t-location scale distribution fitting results are closer to the frequency histograms.

To quantitatively compare the distribution function fitted the definition of Fit Index:

$$
\begin{aligned}
& I=\sum_{i=1}^{M}\left(y_{i}-\bar{N}_{i}\right)^{2} \\
& y_{i}=f\left(\bar{C}_{i}\right)
\end{aligned}
$$

Table 2 Fitting error indicators

\begin{tabular}{cc}
\hline Distribution & Fitting error indicators \\
\hline t-location scale Distribution & 0.000526 \\
Non-parametric Distribution & 0.000555 \\
Normal Distribution & 0.000944 \\
Logistic Distribution & 0.0013327 \\
Extreme Distribution & 0.0092290 \\
\hline
\end{tabular}

Best probability analysis above are derived electrical and mechanical wind power fluctuations unit from qualitative and quantitative point of view distribution.

\section{Distributions.}

The probability of wind power fluctuations compared of different units at different times. For different units, analysis of different periods need to control variables. A first fixed units, respectively, the probability of obtaining it in 30 days in the daily distribution. Then fixed a day, five units were to 
see this day in the probability distribution of power. Due to the amount of data too, here only for one unit of 1 to 30, and the date when the case of all 28 units for comparison. Other cases in the appendix.

Different units in the same period of probability distributions:

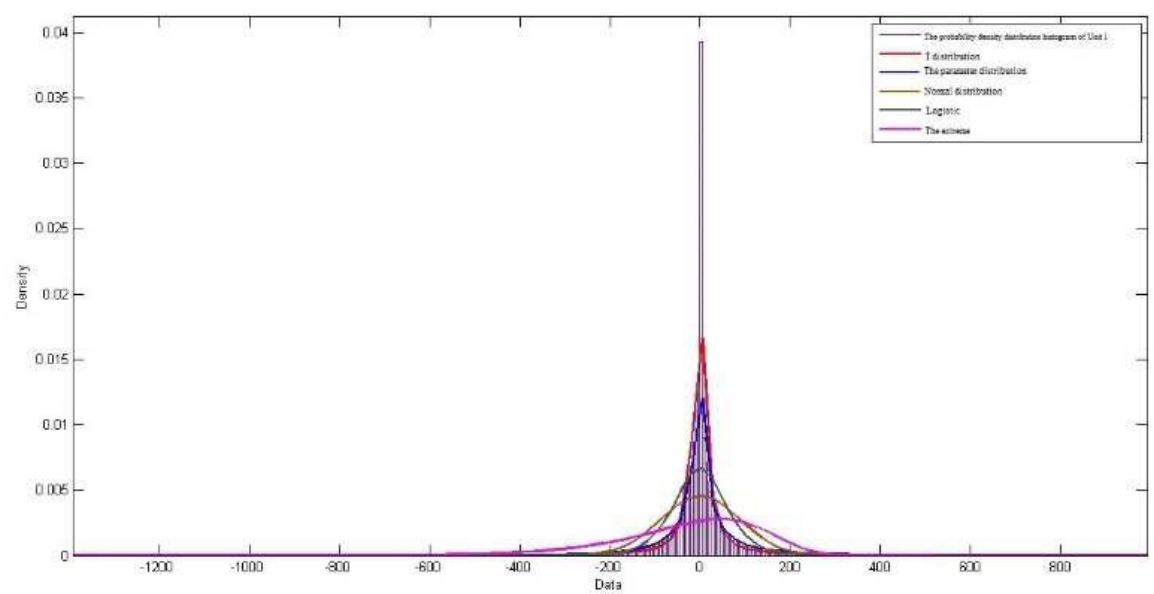

Fig.1 Wind Power Distribution Probability Density Diagram

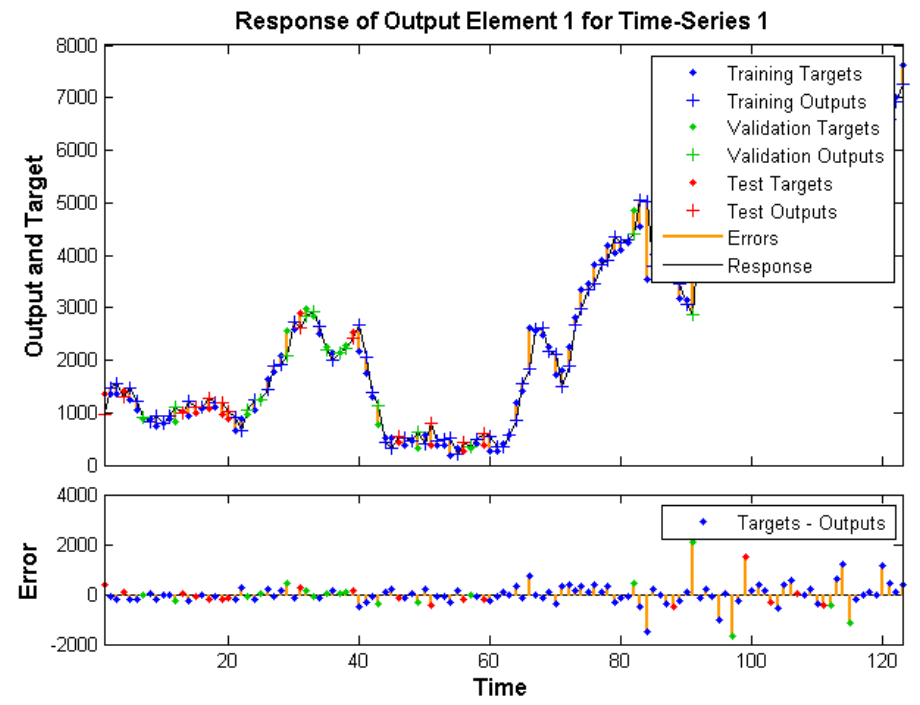

Fig.2 Response of Output Element 1 for Time-Series 1

\section{Conclusion}

Based on m-level wind power fluctuations slip differential calculations and wind power by wavelet neural prediction, a comprehensive analysis of the characteristics of wind power fluctuations. Making the following conclusions:

1) A wind turbine power' output and distribution probability of its fluctuation quantity under different time scale is different, and the distribution of probability increases with sampling interval of the above characteristics of increasing trends.

2) When large-scale wind power connected to the power grid, the power fluctuation may exceed the regulation of power grid capacity and was forced to abandon the wind. This not only reduces the utilization efficiency of wind power equipment, also limits the development of wind power scale.

\section{References}

[1] Yang Cui, Gang Mu, Yu Liu, G. g Yan. Spatiotemporal Distribution Characteristic of Wind Power Fluctuation. North China Electric Power University, Baoding. Power System Technology, 2011, 35(2), p. 110-114. 
[2] Lin satellite, Wen Jin $\mathrm{Yu}$, Meng Ai small, and so the probability of wind power fluctuation characteristics of distribution of [J] Chinese CSEE, 2012, 32 (1): 38-46.

[3] G Browns word R, Kariniotakis G, et al. The state-of-the-art in short-term prediction of wind power: A literature overview[R]. ANEMOS. 2011.

[4] Wang Li jie, Dong Lei, Liao Chung, and other forecasting based on wavelet analysis of short-term wind farm generated power [J] Chinese CSEE, 2009 (28): 30-33.

[5] Hong Hai sheng. Applies to stabilize fluctuations in wind power capacity multi-type storage system configuration control and coordination of Zhejiang University, 2013. 